http://dx.doi.org/10.35381/r.k.v6i2.1243

\title{
Propuesta metodológica adaptada a estudiantes con discapacidad visual en Educación Física
}

Methodological proposal adapted to students with visual disabilities in Physical Education

\author{
Pedro Vicente Hurtado-Guamán \\ pedro.hurtado@est.ucacue.edu.ec \\ Universidad Católica de Cuenca, Azogues \\ Ecuador \\ https://orcid.org/0000-0002-1776-5996 \\ Wilson Hernando Bravo-Navarro \\ wilson.bravo@ucacue.edu.ec \\ Universidad Católica de Cuenca, Azogues \\ Ecuador \\ https://orcid.org/0000-0003-3381-8063
}

Recepción: 25 de enero 2021

Revisado: 20 de febrero 2021

Aprobación: 30 de abril 2021

Publicación: 15 de mayo 2021 


\title{
RESUMEN
}

El objetivo de la presente investigación fue determinar las adaptaciones metodológicas a estudiantes con discapacidad visual en las clases de Educación Física, se efectuó a través de un diseño no experimental, con un alcance descriptivo de corte transversal, tomando una muestra de 43 docentes de Educación Física de las ciudades de Cuenca y Azogues, de los cuales 15 son mujeres y 28 varones, se manejó un muestreo no probabilístico por conveniencia, se utilizó la técnica de la encuesta, misma que fue validad por expertos. Se encontró que los docentes de Educación Física presentan un desconocimiento acerca de las metodologías y adaptaciones que se deben implementar en las clases para los estudiantes con discapacidad visual, además existe un déficit en cuanto a; infraestructura accesible, material adaptado, metodologías apropiadas, ya sea por desconocimiento, falta de aplicación de leyes y poca capacitación.

Descriptores: Experimento educacional; educación de ciegos; educación física. (Palabras tomadas del Tesauro UNESCO).

\begin{abstract}
The aim of this research was related to determine the methodological adaptations of students with visual disabilities in Physical Education classes, it was carried out through a non-experimental design, with a descriptive cross-sectional methodological scope, taking a sample of 43 Physical Education teachers from the cities of Cuenca and Azogues, in these sample, 15 were women and 28 were men, a non-probabilistic convenience sampling was used, the survey technique was used to develop this research, the survey was validated by experts. It was found that Physical Education teachers had a lack of knowledge about the methodologies and adaptations and a new structure of knowledge should be implemented in classes for students with visual disabilities, there is also a deficit in terms of; accessible infrastructure, adapted material, appropriate methodologies, either due to ignorance, lack of new application topics to change this reality into an inclusive kind of education.
\end{abstract}

Descriptors: Educational experiments; education of the blind; physical education. (Words taken from the UNESCO Thesaurus). 


\section{INTRODUCCIÓN}

En la actualidad los establecimientos educativos están sujetos a los tratados internacionales, a la constitución nacional y demás leyes de la República del Ecuador, con respecto a las personas con discapacidad, el numeral 7 del artículo 47 de la Constitución de la República del Ecuador establece que; el estado garantizará políticas de prevención de las discapacidades y, de manera conjunta con la sociedad y la familia, procurará la equiparación de oportunidades para las personas con discapacidad y su integración social.

Se reconoce a las personas con discapacidad, los derechos a una educación que desarrolle sus potencialidades y habilidades para su integración y participación en igualdad de condiciones. Se garantizará su educación dentro de la educación regular. Los planteles regulares incorporarán trato diferenciado y los de atención especial a la educación especializada. Los establecimientos educativos cumplirán normas de accesibilidad para personas con discapacidad (Asamblea Nacional del Ecuador, Constitución de la República del Ecuador, 2008).

Lamentablemente sucede en la teoría, pero no hay procesos adaptados a la práctica, donde el docente socialice un plano real de equidad, para que verdaderamente se dé un proceso de inclusión con metodologías pedagógicas y actividades adaptadas. La Educación Física es un derecho en el que todos los estudiantes deben disfrutar, sin embargo, dentro de la comunidad educativa, no garantiza la participación de todos los estudiantes, ya sea por problemas económicos, sociales o la falta de conocimiento de los docentes de Educación Física, sin permitir que los estudiantes adquieran y demuestren sus habilidades y destrezas (Samomons \& Bakkum, 2011).

Es necesario para los estudiantes participar de la Educación Física, actividad física, deporte y recreación adaptada a sus necesidades, por lo tanto, es necesario motivar y sensibilizar a los profesores y compañeros, con las metodologías adaptadas para la práctica de educación física (Rodríguez-Bies, 2015).

La escuela tiene la misión de formar, socializar, incluir de manera integral, sin embargo, muchas veces por la falta de formación adecuada podría ocasionar desinterés y un abierto rechazo hacia la incorporación de alumnos con necesidades educativas especiales a la educación. En tanto, la sociedad debe centrarse en 
motivar a practicar dentro del establecimiento y fuera del mismo, por los múltiples beneficios que aportan a los seres humanos, por ejemplo; evita el sedentarismo, problemas cardiacos, mejora la autoestima, evita el sobrepeso y obesidad, entre otros, estos son obviamente para toda la población.

A través de la práctica y la sensibilización con las metodologías adaptadas, se buscará una verdadera inclusión educativa, siempre y cuando las propuestas metodológicas adaptadas puedan conseguir que los procesos y aprendizajes sean de manera significativas, proporcionando bienestar y salud (Fernandez, et al. 2016).

\section{Referencial teórico}

En el presente artículo, se aborda la metodología a seguir en la enseñanza de diversas tareas útiles para el docente de Educación Física (Arráez, 2008), dotando al docente de numerosas estrategias didácticas válidas para aplicar dentro del aula. En esta ocasión se analizará en un supuesto práctico destinado a la presencia de 43 docentes de diferentes instituciones educativas de las ciudades de Azogues y Cuenca pertenecientes a las provincias de Cañar y Azuay respectivamente.

Para (Pastor-Vicente, 2006), la educación inclusiva ha evolucionado a lo largo de los años, para ello han influenciado; cambios políticos, sociales, educativos, tecnológicos, médicos, psicológicos y pedagógicos, mismos que siguen en constante evolución, dado que es un ámbito de estudio relativamente joven. En Ecuador, se fomenta la inclusión educativa desde el año 2012, donde empieza a regir la ley orgánica de discapacidades, aquí empieza la implementación de medidas para la inclusión educativa de los estudiantes con discapacidad que requieran apoyo técnico, tecnológico y humano, además de las adaptaciones curriculares, accesibilidad, y equipos de comunicación (Asamblea Nacional del Ecuador, Ley Organica de Discapacidades, 2012).

(Aguado, et al. 2008), comenta que el lugar del trabajo en el que se encuentra el profesor debe garantizar al estudiante con discapacidad una comodidad ya sea en el aula $o$ en el patio, para que se sienta con seguridad, así provoca motivación y entusiasmo. Para (Lopez-Melero, 2011) gracias al movimiento las personas con 
discapacidad visual pueden movilizarse de un lugar a otro, facilitando el desplazamiento, siempre y cuando no exista barreras que impidan la movilidad.

Las personas con discapacidad logran adaptarse al medio en el que los rodea, produciéndose un mapa mental, además el oído y el tacto son los órganos que facilitan la movilidad de las personas que padecen esta enfermedad, la secuencia de aprendizaje de la persona ciega es muy diferente a las demás personas (Sanz-Rivas \& Reina-Vaillo, 2012).

Siempre se debe intentar que los estudiantes con discapacidad sean incluidos de una manera efectiva y afectiva, ya que se trata de seres humanos que sienten y perciben las percepciones, sensaciones y motivaciones que los rodean (Aguirre-Barco, 2008). (Fernández-Batanero, 2008) Las adaptaciones curriculares es el paso para una buena inclusión educativa, en especial de los estudiantes con discapacidad visual, luego generando las adaptaciones a los materiales que se utilizan en las clases de educación física. Según (Lieberman, 2010). El profesor, los compañeros, y los familiares son los pilares en la educación, de tal forma que se convierten luego en motivación personal y cada día se pondrá de manifiesto para ir superando los desafíos.

De acuerdo con (Loeckemeyer, 2006) utilizar estrategias y recursos didácticos durante las clases de Educación Física inclusiva puede ayudar a conseguir el éxito en el aprendizaje de las habilidades motrices, fomentar la autonomía, la auto-superación, aceptar y valorar su propio cuerpo. Estas consideraciones deben ser tomadas en cuenta antes de iniciar, durante y después de una clase de Educación Física.

(Cumellas, et al. 2006). Al inicio de cada periodo lectivo es necesario disponer del certificado médico para saber cuál es su diagnóstico, que presenta el estudiante, así se puede tener en cuenta si el estudiante presenta contraindicaciones para el movimiento, así mismo, al principio de la sesión, se debe comunicar anticipadamente que se va a realizar en ese día, y así el estudiante está preparado mentalmente, por otro lado, cuando se dirige al estudiante decirle por su nombre en voz alta para que lo pueda escuchar, también se lo puede apoyar de su brazo, o en otros casos para que se apoye darle el hombro (Rosa-Guillamón \& García-Cantó, 2018) 
Siempre hay que dirigirse de frente al estudiante para crear interés en el mismo, los mensajes deben ser claros y precisos, este es un factor muy importante que puede motivar o desmotivar al estudiante, además siempre se debe consultar si lo comprende, caso contrario utilizar otro lenguaje hasta que el estudiante logre comprender lo que está realizando.

La comunicación debe ser concreta y fácil, siempre las indicaciones deben ser verbales así mismo, otro medio importante que se debe procurar utilizar es el tacto, y las indicaciones deben partir desde lo específico hacia lo general, para una buena comprensión. Por otro lado, la creación de códigos verbales entre el estudiante, los compañeros y el profesor es muy importante esto ayuda mucho, ya que el estudiante tiene seguridad a la hora de la práctica (Sapon-Shevin, 2013).

Después de las instrucciones es recomendable que un compañero lo asista para apoyarle en la ejecución de la actividad que va a realizar, luego preguntarle cómo se siente después de ejecutar el ejercicio y si es posible pedir que siga repitiendo varias veces, esto hace que se sienta seguro, otro punto muy importante es procurar no asustarle.

El espacio en donde se va a realizar las actividades, el estudiante con discapacidad debe realizar un previo reconocimiento, para que esté orientado en el espacio y las cosas que lo rodean, este lugar debe tener por lo menos líneas táctiles, los materiales deben ser sonoros, así mismo estos materiales tienen que estar al alcance y siempre en el mismo lugar, para evitar que los estudiantes con discapacidad se pierdan en el espacio, en otras palabras los materiales, y la infraestructura deben estar adaptados, para evitar accidentes, procurando que el estudiante tenga una verdadera inclusión. (Aguilera-Garcia , 2016).

\section{MÉTODO}

La presente investigación se caracteriza por ser de un un diseño no experimental, con un alcance descriptivo de corte transversal, aplicando el método deductivo, para el proceso de investigación, se utilizó la técnica de la encuesta y como instrumento un cuestionario de preguntas validado por expertos en la temática. La población son los docentes de Educación Física de las ciudades de Cuenca y Azogues, para la selección 
de la muestra se empleó un muestreo no probabilístico por conveniencia, quedando conformada de 43 docentes de Educación Física de las ciudades de Azogues (22) y Cuenca (21) de las cuales 15 son mujeres y 28 son varones (Prado-Prado, et al. 2020). Luego de la validación del cuestionario de preguntas, se realiza el diseño del cuestionario en la aplicación google forms, seguidamente la recolección de datos, para esto se utiliza las distintas redes sociales y demás herramientas digitales disponibles en la actualidad.

Para realizar el análisis estadístico se hizo el uso de la herramienta SPSS versión 25 con el objetivo de obtener frecuencias, la media y porcentajes de las variables socio demográfico, además de los valores de la desviación estándar de cada una de las categorías que conforman el cuestionario de preguntas

\section{RESULTADOS}

Tabla 1.

Promedio de Edad de los docentes de Educación Física.

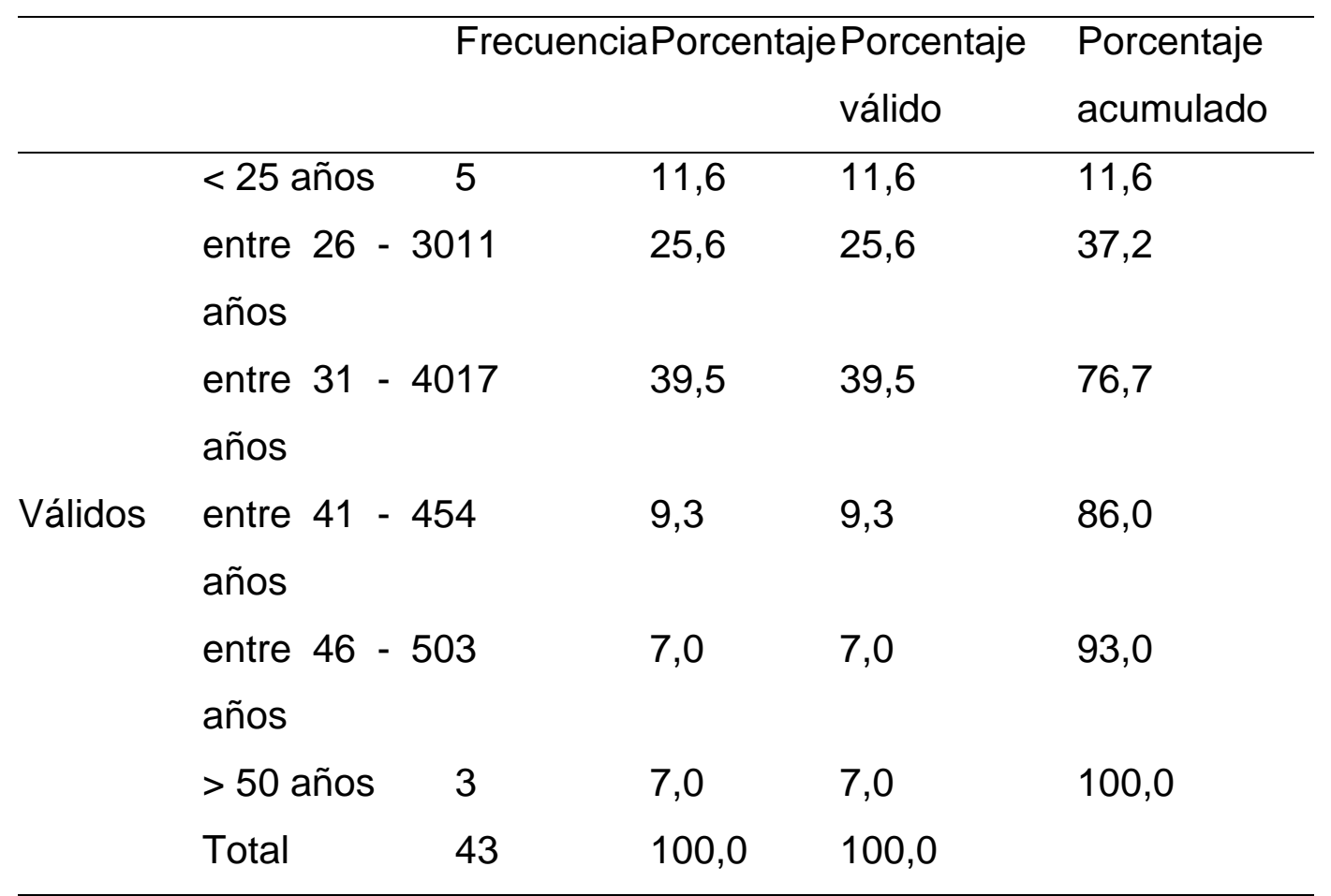

Fuente: Encuesta. 
Del total de los 43 encuestados se ha notado que la edad de más participación es de entre los 31 y 40 años, esto siendo demostrado con un porcentaje válido del 39,5\%, el $25,60 \%$ consta de una edad de 26 a 30 años, lo que demuestra que esta frecuencia indica un alce del $76,7 \%$ acumulado.

\section{Tabla 2.}

¿Trabaja Ud. frecuentemente con estudiantes con discapacidad visual en el área de Educación Física?

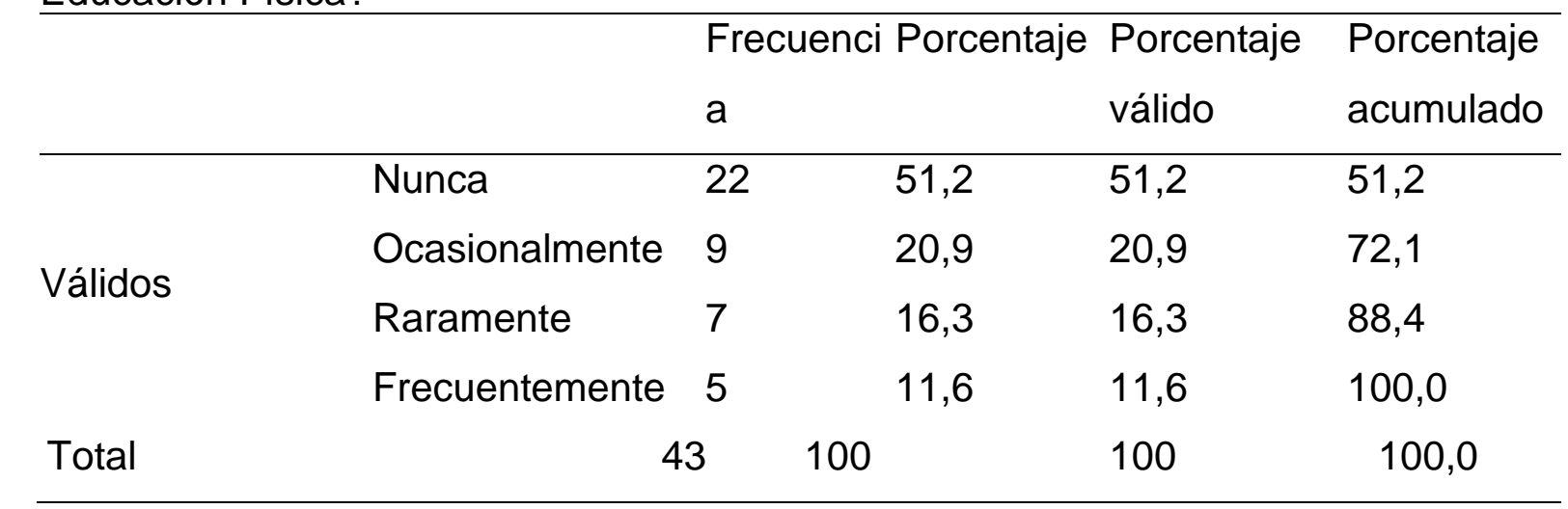

Fuente: Encuesta.

El $51,2 \%$ de los docentes del área de Educación Física indican que nunca han trabajado ni de manera frecuente, así como de ninguna manera con estudiantes con ningún tipo de discapacidad de tipo visual.

Tabla 3 
¿Durante el tiempo en que Ud. ha venido laborando ha recibido capacitación acerca de las adaptaciones para estudiantes con discapacidad visual?

\begin{tabular}{|c|c|c|c|c|}
\hline & \multicolumn{3}{|c|}{ Frecuen PorcentajePorcentaje } & \multirow{2}{*}{$\begin{array}{l}\text { Porcentaje } \\
\text { acumulado }\end{array}$} \\
\hline & cia & & válido & \\
\hline No & 32 & 74,4 & 74,4 & 74,4 \\
\hline Válidos Si & 11 & 25,6 & 25,6 & 100,0 \\
\hline Total & 43 & 100,0 & 100,0 & \\
\hline
\end{tabular}

Fuente: Encuesta.

Los docentes afirman que en el transcurso del tiempo que han trabajado como docentes de Educación Física, no han recibido ningún tipo de capacitación para impartir las clases a estudiantes con discapacidad visual. Esto se demuestra con el $74,4 \%$ que indican dicha versión, pero el $25,60 \%$ si han recibido la capacitación necesaria.

Tabla 4 ¿Conoce Ud. ¿Los materiales que necesitan los estudiantes con discapacidad visual en una clase de Educación Física?

\begin{tabular}{|c|c|c|c|c|c|}
\hline & \multirow{2}{*}{$\begin{array}{l}\text { Frecue } \\
\text { ncia }\end{array}$} & \multicolumn{2}{|c|}{ Porcentaj Porcentaje } & \multirow{2}{*}{$\begin{array}{l}\text { Porcentaje } \\
\text { acumulado }\end{array}$} \\
\hline & & & e & válido & \\
\hline \multirow{4}{*}{ Válidos } & Poco & 29 & 67,4 & 67,4 & 67,4 \\
\hline & Nada & 12 & 27,9 & 27,9 & 95,3 \\
\hline & Mucho & 2 & 4,7 & 4,7 & 100,0 \\
\hline & Total & 43 & 100,0 & 100,0 & \\
\hline
\end{tabular}

Fuente: Encuesta.

Se calcula que el $67,4 \%$ como porcentaje válido indican conocen poco los materiales respectivos para los estudiantes con discapacidad visual, por consecuente se conoce 
que el $27,9 \%$ no conoce nada de estos materiales para el uso en las clases de Educación Física.

Tabla 5.

¿Conoce Ud. las metodologías de enseñanza en el área de Educación Física para estudiantes con discapacidad visual?

\begin{tabular}{clllll}
\hline & \multicolumn{2}{l}{$\begin{array}{l}\text { Frecuen Porcentaje } \\
\text { cia }\end{array}$} & $\begin{array}{l}\text { Porcentaje } \\
\text { válido }\end{array}$ & $\begin{array}{l}\text { Porcentaje } \\
\text { acumulado }\end{array}$ \\
\hline \multirow{2}{*}{ Válidos } & Poco & 30 & 69,8 & 69,8 & 69,8 \\
& Nada & 11 & 25,6 & 25,6 & 95,3 \\
\cline { 2 - 3 } & 2 & 4,7 & 4,7 & 100,0 \\
Total & 43 & 100,0 & 100,0 & \\
\hline
\end{tabular}

Fuente: Encuesta.

Poco conocen los docentes encuestados sobre las metodologías de enseñanza en el área de Educación Física para estudiantes con discapacidad visual, esto dando un porcentaje acumulado muy notorio como es del 69,8\%, demostrándose que los docentes conocen un porcentaje mínimo del 4,7\% mucho sobre estas metodologías en cuestión.

\section{Tabla 6.}


Desviación Estándar.

\section{Estadísticos}

Edad ¿Trabaja Ud.¿La ¿Durante elConoce Ud.¿Conoce Ud. frecuenteme institución tiempo en que ¿Los las nte condonde Ud.Ud. ha venidomateriales metodologías estudiantes trabaja laborando haque de con cuenta con larecibido necesitan enseñanza discapacidadinfraestructur capacitación los en el área de visual en ela accesibleacerca de lasestudiantes Educación área depara adaptaciones con Física para Educación estudiantes para discapacida estudiantes Física? con estudiantes $d$ visual encon discapacidadcon una clasediscapacidad visual? discapacidad de visual? visual? Educación

Física?

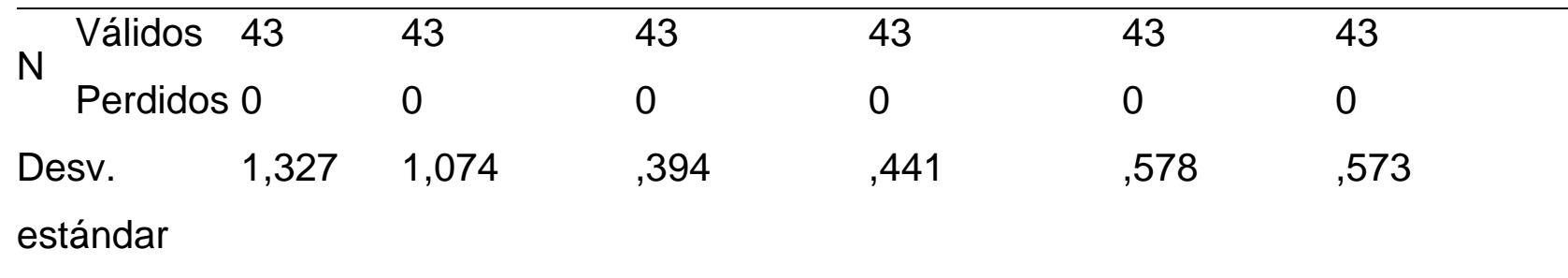

Fuente: Encuesta.

El profesorado muestra una desviación del 0,573 en cuanto al conocimiento de las metodologías de enseñanza dentro del área de Educación Física. De acuerdo al 
objetivo planteado en determinar las adaptaciones metodológicas en estudiantes con discapacidad visual y con los resultados obtenidos en la accesibilidad, adaptaciones en los materiales, adaptaciones curriculares, se puede entender que, en la actualidad, el reto más grande de los profesores del área de Educación Física es trabajar mediante la inclusión educativa efectiva.

La educación inclusiva es responsabilidad de los docentes del correcto aprendizaje, que buscan las mejores decisiones para garantizar la equidad y a su vez un buen rendimiento por parte de los estudiantes (Pinilla, 2011). Por otro lado, en los resultados sobre la accesibilidad, se puede manifestar que el estado tiene que brindar instituciones educativas accesibles, ya que en la actualidad no sucede, y para esto nos confirma el resultado de la encuesta realizada a los docentes de educación física. Así mismo, en Europa como, por ejemplo, en la capital de España el gobierno local construyo escenarios deportivos accesibles por toda la ciudad, además de implementar de materiales adaptados, para que todos puedan realizar algún tipo de ejercicio o participe de algún juego (Comunidad Madrid, 2018). En tanto que los profesores, según los resultados obtenidos requieren de una amplia capacitación en la prestación del servicio educativo a este grupo de atención prioritaria.

Los problemas de formación de los docentes, en el sistema educativo, es aquel docente limitado en las metodologías apropiadas, creando dificultades cuando se trata de realizar adaptaciones curriculares para estudiantes que tengan alguna discapacidad (Luque-Parra \& Luque-Rojas, 2011). Otro punto muy importante es realizar las adaptaciones apropiadas en los materiales, para que la enseñanza sea inclusiva y los estudiantes con discapacidad visual logren una educación integral.

Las adaptaciones pueden ser en los elementos personales, materiales, organizativos, de comunicación y así se garantiza Una metodología correcta, esto hace que los docentes realicen su trabajo con eficiencia, mientras los estudiantes gozan de un aprendizaje significativo, y gocen de una verdadera inclusión educativa. Las adaptaciones curriculares son un factor importante para llevar a cabo una verdadera inclusión, motivo por el cual el rol de un docente está en la aplicación diaria de las adaptaciones, fomentando desde una sesión de clase, el compañerismo, y demás valores que es muy importante (Aquino-Zúñiga, et al. 2012). 
La modificación del currículo, se puede realizar sin perder de vista, la manera significativa de aquellos estándares de aprendizaje, indicadores de logro, instrumentos de evaluación, modificación de contenidos, metodología, actividades, evaluaciones.

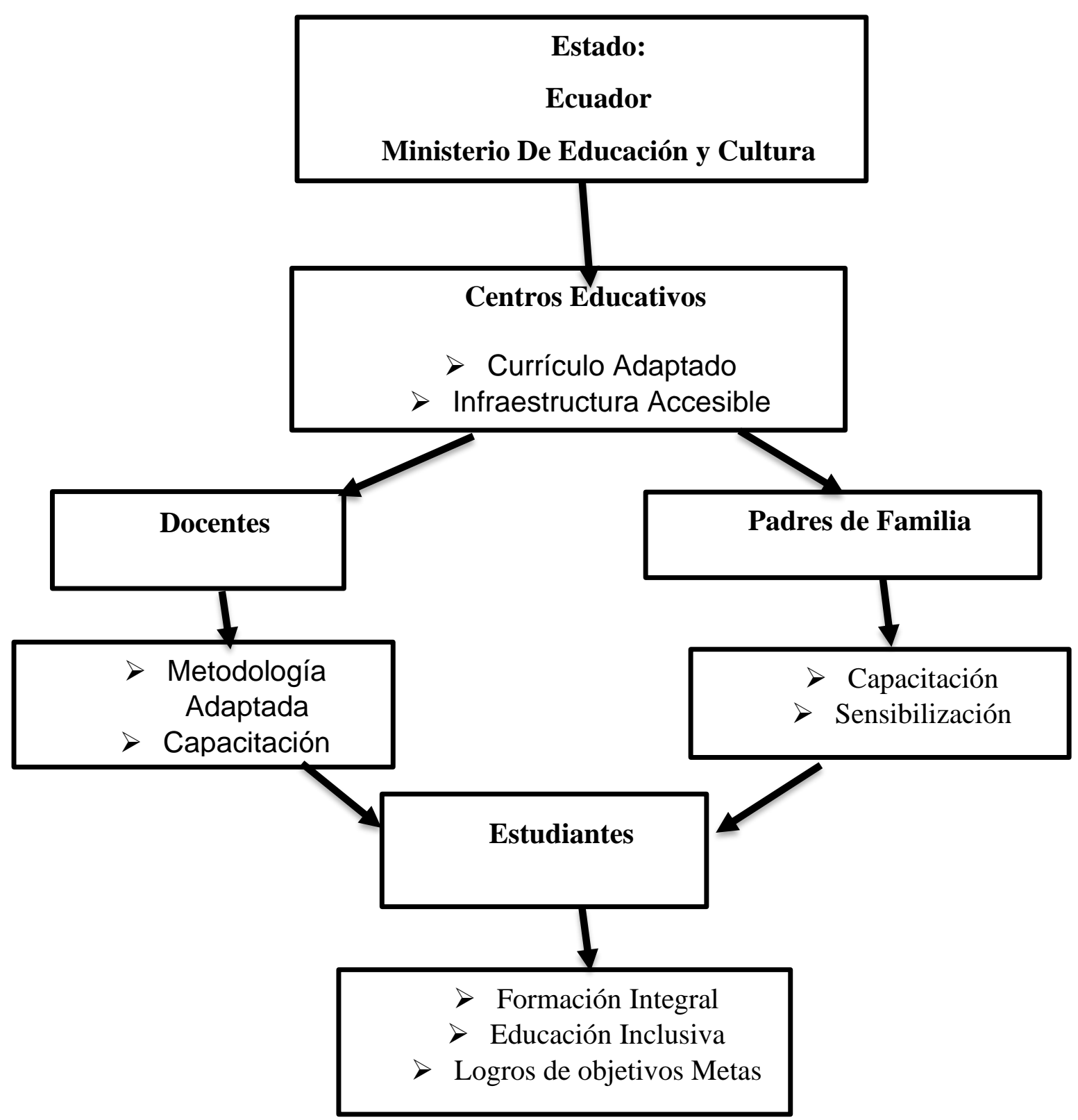

Figura 1. Esquema de la propuesta.

Fuente: Los Autores (2021).

En el Ecuador se tendría que implementar en los centros educativos con la infraestructura accesible, dotar de los materiales adaptados, capacitar a los docentes 
en las metodologías apropiadas en el área de Educación Física, sensibilizar a la sociedad en general, para que exista una verdadera inclusión.

La infraestructura debería ser accesible en todos los centros educativos, sin embargo, según este estudio realizado en las dos ciudades, no existen centros educativos accesibles, los estudiantes con discapacidad visual requieren de señaléticas donde se pueda movilizar sin miedo o temor de sufrir un accidente.

Los padres de familia y familiares también deberían recibir capacitaciones para apoyar a su familiar con discapacidad visual, dejando a lado la sobre protección o el abandono en algunos casos, muchas veces los padres, o familiares no conocen la realidad de un estudiante con discapacidad visual, en vez de ayudarlo se convierte en una frustración, es ahí que la capacitación, sensibilización juega un rol muy importante.

En Educación Física se debe utilizar metodologías apropiadas que puedan solucionar todas las necesidades de todos los estudiantes, para esto se recomienda realizar, un plan de actividades adaptadas a la realidad.

Se debería trabajar mediante actividades colectivas que favorecen en el estudiantado; la participación, la resolución de problemas y la relación con los demás estudiantes de la institución educativa que son los protagonistas para la inclusión verdadera de los estudiantes con discapacidad visual en la sociedad.

\section{CONCLUSIONES}

Los docentes del área de Educación Física presentan un claro desconocimiento sobre las metodologías y adaptaciones que se deben implementar en las clases, para los estudiantes con discapacidad visual, por tal motivo es necesario la capacitación y actualización de los conocimientos en estos aspectos.

Es obligación del estado, la dotación de infraestructura accesible, materiales adaptados, programas de capacitación masiva, aplicación de las leyes, que se encuentran vigentes, ya que en esta investigación se demuestra que existe un déficit de lo que se ha mencionado anteriormente.

La Educación Física busca la armonía en el desarrollo de los estudiantes con discapacidad, por esta razón las instituciones educativas tienen que dotar de las 
herramientas necesarias a los docentes, para que puedan los estudiantes gozar de una verdadera inclusión educativa.

\section{FINANCIAMIENTO}

No monetario.

\section{AGRADECIMIENTO}

A la Universidad Católica de Cuenca y la Jefatura de Posgrados por apoyar el desarrollo de la investigación.

\section{REFERENCIAS CONSULTADAS}

Aguado, A. L. Flórez, M. A. \& Alcedo, M. A. (2004). Programas de cambio de actitudes ante la discapacidad [Programs to change attitudes towards disability]. Psicothema 76 (4). 667-673.

Aguilera-Garcia , J. (2016). Actitudes hacia la discapacidad de aspirantes a educadores sociales. propuestas de formación para una educación inclusiva [Attitudes towards disability in students of Degree of Social Education. Proposals for Inclusive Education]. Revista nacional e internacional de educación inclusiva, 9(1), 13-29.

Aguirre-Barco, P. et al. (2008). Manual de atención al alumnado con necesidades especificas de aoyo educativoderivadasde discapacidad visual y sordicegue Manual de atención al alumnado con necesidades especificas de aoyo educativoderivadasde discapacidad visual y sordiceguera [Manual of attention to students with specific educational support needs derived from visual disability and deafblindness Manual of attention to students with specific educational support needs derived from visual disability and deafblindness]. http://hdl.handle.net/11162/3177

Aquino-Zúñiga, S, García-Martínez, V, \& Izquierdo, J. (2012). La inclusión educativa de ciegos y baja visión en el nivel superior: Un estudio de caso [Educational Inclusion of the Blind and Low Vision at Higher Level: A Case Study]. Sinéctica, (39), 01-21.

Arráez , J. M. (2008). ¿Puedo jugar yo?. El juego modificado propuesta para la integracion de niños y niñas con necesidedes educativas especiales [Can I 
play? The modified game proposed for the integration of boys and girls with special educational needs]. https://n9.cl//knr0

Asamblea Nacional del Ecuador. (2008). Constitución de la República del Ecuador [Constitution of the Republic of Ecuador]. https://n9.cl/sia

Asamblea Nacional del Ecuador. (2012). Ley Organica de Discapacidades. Recuperado de https://n9.cl/zxfi

Comunidad Madrid . (2018). Plan para fomentar la Actividad Física y el Deporte Inclusivo [Plan to promote Physical Activity and Inclusive Sports]. https://n9.cl/ep0ck

Cumellas Riera, M., \& Estrany Font, C. (2006). Juegos convencionales adaptados. En Cumellas Riera, M., Discapacidades motoras y sensoriales en primaria. La inclusión del alumnado en Educación Física. (pp 55-190). Zaragoza: Inde Publicaciones.

Fernandez, J. Lopez, Paz,J: Nabarro Paton , R. (2016). La exprecion corporal orientada al alumnado con discapacidad visual desde una perpectiva inclusiva de la didactica de la educacion fisica [Body expression aimed at students with visual disabilities from an inclusive perspective of the didactic of physical education]. http://hdl.handle.net/10347/18084

Fernández-Batanero, J. (2008). La investigación en educación especial. Líneas temáticas y perspectivas de futuro [The researh in special education. Thematic lines and perspectives]. Perfiles educativos, 30(119), 7-32.

Lieberman, L, J. (2010). Visual Impaiment. En Winnick J. Adapted physicaleducation and New York.

Loeckemeyer, M. (2006). Acercamiento del discapacitado visual al deporte. ¿Porqué y para qué? [Approach of the visually impaired to sport. Why and for what?]. Comunidad Deportiva (1), 1-3.

López-Melero, M. (2012). Barreras que impiden la escuela inclusiva y algunas estrategias para construir una escuela sin exclusiones [Barriers that prevent inclusive school and some strategies to build a school without exclusions]. Innovación Educativa, (21).

Luque-Parra, \& Luque-Rojas, M. J. (2011). Conocimientode la Discapacidad y Relaciones sociales en el aula Inclusiva [Knowledge of Disability and Social 
Relations in the Inclusive Classroom]. Revista Iberoamericana de Educación, 54(6). https://doi.org/10.35362/rie5461640

Pastor-Vicente, C. (2006). Los educadores y las educadoras sociales en el campo de las discapacidades [Educators and social educators in the field of disabilities]. https://n9.cl/2nsc6b

Pinilla, A. (2011). Minusvalías motoras, psíquicas y sensoriales en el desarrollo curricular de la Educación física [Motor, mental and sensory disabilities in the curriculum development of Physical Education]. Revista Paiderex, 60-66.

Prado-Prado, S., García-Herrera, D., Erazo-Álvarez, J., \& Narváez-Zurita, C. (2020). Google Classroom: educational application as a learning environment in rural areas in contexts of COVID-19 [Google Classroom: educational application as a learning environment in rural areas in contexts of COVID-19]. Revista Arbitrada Interdisciplinaria Koinonía, 5(5), 4-26. http://dx.doi.org/10.35381/r.k.v5i5.1031

Rodríguez-Bies, E. (2015). Actividad física y discapacidad sensorial visual: una propuesta didáctica con estudiantes del Grado en Ciencias de la Actividad Física y el Deporte [Physical activity and visual sensory disability: a didactic proposal with students of the Degree in Physical Activity and Sports Sciences]. Arte Y Movimiento, (13).

Rosa-Guillamón, A., \& García-Cantó, E. (2018). Adaptaciones en el área de Educación Física: propuestas prácticas para las discapacidades visual y auditiva [Adaptations in the area of Physical Education: practical proposals for visual and auditory disabilities]. Lecturas: Educación Física Y Deportes, 23(246), 102122.

Samomons, P. \& Bakkum, L. (2011). Escyelas eficaces, equidad y eficaica docente: una revision de la literatura. Profesirado [Effective Schools, Equity and Teacher Effectiveness]. Profesorado. Revista de curiculum y formación del profesorado, 15(3).

Sanz-Rivas D. Reina-Vaillo, R. (2012). Acrividades fisicas fisicas y deportes adaptados para personas con discapacidad [Physical physical activities and sports adapted for people with disabilities]. Badalona. 
Sapon-Shevin, M. (2013). La inclusión real: Una perspectiva de justicia social [versión en castellano]. Revista De Investigación En Educación, 11(3), 71-85.

(C2021 por los autores. Este artículo es de acceso abierto y distribuido según los términos y condiciones de la licencia Creative Commons Atribución-NoComercial-Compartirlgual 4.0 Internacional (CC BY-NC-SA 4.0) (https://creativecommons.org/licenses/by-nc-sa/4.0/). 